\title{
Management of hepatic artery aneurysms
}

\author{
Polat $E^{1}$, Ozogul $\mathrm{YB}^{1}$, Ercan $\mathrm{M}^{1}$, Karaman $\mathrm{K}^{1}$, Aksoy $\mathrm{E}^{1}$, Kucukay $\mathrm{F}^{2}$, Surmelioglu $\mathrm{A}^{1}$, Dalgic $\mathrm{T}^{1}$, \\ Ulas $\mathrm{M}^{1}$, Bostanci $\mathrm{EB}^{3}$, Akoglu $\mathrm{M}^{3}$
}

Turkiye Yuksek Ihtisas Teaching and Research Hospital, Department of Gastroenterological Surgery, Ankara, Turkey. keremkaraman1972@yahoo.com

\begin{abstract}
Background: Hepatic artery aneurysm (HAA) is a rare clinical entity that can lead to potentially life threatening complications. We reported our personal experience of 4 cases, in which we used different procedures. Methods: The first case had a pseudo-aneurysm involving the right hepatic artery. The second case had a pseudoaneurysm, which was localized distal to the accidentally ligated right hepatic artery from the previous cholecystectomy operation. The third case had multiple aneurysms with accompanying dissecting abdominal aortic aneurysm. The fourth case had a pseudo-aneurysm originating from the proper hepatic artery. A covered stent was successfully placed in the case 1. In the second case, the right hepatic artery was ligated distal to the aneurysm. In the third case, vascular structures were not appropriate for vascular reconstruction, and a covered stent placement and embolization were unsuccessful. In the fourth case, ligation of the proper hepatic artery and cholecystectomy was performed. Results: The third case with multiple aneurysms died from multi-organ failure due to sepsis. The remaining cases (case 1,2, and 4) are disease free and alive.

Conclusion: HAAs are more commonly observed clinical entities, and their treatment should be handled for each patient separately. Computerized tomography-Angiography and intraoperative Doppler ultrasound are useful radio-diagnostics for determination of aneurysm and planning the operative procedure (Fig. 5, Ref. 15). Full Text in PDF www.elis.sk. Key words: pseudo-aneurysm, covered stent, embolization, angiography.
\end{abstract}

Hepatic artery aneurysm (HAA) is a rare clinical entity that can lead to potentially life threatening complications when it ruptures. Among visceral artery aneurysms, HAAs are the second most common site after splenic artery aneurysms and make up 12-20\% of them. The most frequently associated condition with HAA is atherosclerosis. Hypertension is the most common co-morbid disease followed by malignancy, peptic ulcer disease, peripheral artery disease, obesity and chronic obstructive pulmonary disease. There seems to be a strong association with tobacco and alcohol consumption (1).

The first surgical approach to treat HAA was ligation of the proper hepatic artery by Kehr in 1903 (2). Since then, many other treatment options evolved such as transarterial embolization, covered stent placement, resection of the aneurysm with or without vascular reconstruction, arterial by-pass and hepatectomy.

HAA's have become more commonplace. This change likely reflects the increased use of percutaneous diagnostic and therapeutic biliary procedures, as well as increased use of diagnostic computed tomography $(C T)$ (3). But, even today, there is still not a standard treatment modality for HAA. We reported our personal experience of 4 cases with HAA, in which we used different procedures and compared our results to literature.

${ }^{1}$ Turkiye Yuksek Ihtisas Teaching and Research Hospital, Department of Gastroenterological Surgery, Ankara, Turkey, ${ }^{2}$ Turkiye Yuksek Ihtisas Teaching and Research Hospital, Department of Radiology, Ankara, Turkey, and ${ }^{3}$ Turkiye Yuksek Ihtisas Teaching and Research Hospital, Department of Gastroenterological Surgery, Ankara, Turkey

Address for correspondence: K. Karaman, MD, Altinoluk sok 12, 10 06010, Etlik, Ankara, Turkey,

Phone: +90.312.3061434, Fax: +90.312 .3124120$

\section{Case reports}

Case 1

A 29-year-old female patient was re-admitted 4 weeks after laparoscopic cholecystectomy $(L C)$ operation for an acute upper gastrointestinal bleeding and persistent jaundice. An emergent upper endoscopy was performed and no primary focus was detected. Hemobilia and benign biliary stricture was found during endoscopic retrograde pancreatico-cholangiography $(E R C P)$ and a nasobiliary drainage catheter $(N B D C)$ was placed simultaneously. Upon the blood transfusions the patient continued to bleeding and an angiography was performed. An isolated pseudo-aneurysm $3 \mathrm{~cm}$ in diameter originating from the right hepatic artery was found. A $5 \times 25 \mathrm{~mm}$ covered stent (Gore Viabahn, USA) was placed successfully to close the aneurysmal neck (Fig. 1). The patient recovered

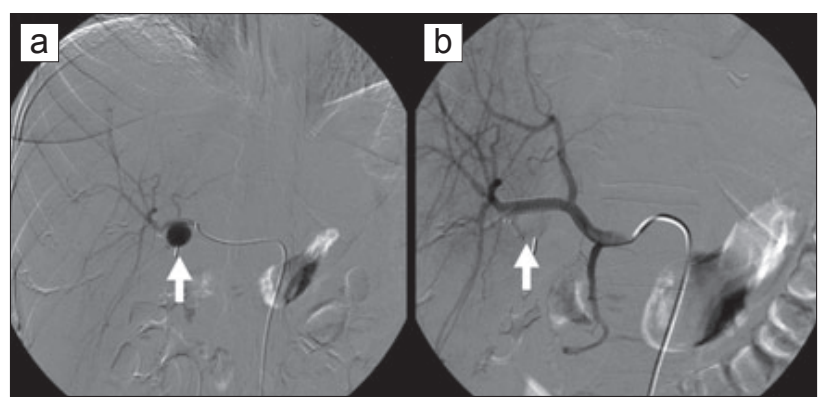

Fig. 1. a) Angiography shows a pseudo-aneurysm from the right hepatic artery. b) A covered stent was successfully placed to close the aneurysmal neck. 
rapidly in the following days, jaundice improved and bleeding stopped. Control angiography showed a patent right hepatic artery with exclusion of the aneurysm. The $N B D C$ was removed and the patient was discharged after her symptoms disappeared and biochemical parameters went down to normal levels.

\section{Case 2}

A 49-year-old male patient was admitted with acute biliary pancreatitis two weeks after a conversion cholecystectomy operation. Under follow up in the clinic with conservative medications cholestatic liver enzymes increased and the patient began to suffer from hematemesis on the twelfth day. No primary focus was detected during upper endoscopy but bleeding from the biliary system trough papilla into the duodenum was seen. The stones in the common bile duct were removed and a $N B D C$ was placed. An angiography was performed and a $4 \times 5 \mathrm{~cm}$ aneurysm was found just distal to the accidentally ligated right hepatic artery from the previous cholecytectomy operation. The aneurysm had multiple collaterals in association with left hepatic and other arteries in the vicinity (Fig. 2). Endovascular covered stent placement was found unsuitable for this patient, because there were multiple tortuous collaterals, which were of inappropriate size for applying the covered stents currently available in the market. Coil embolization was also unsuccessful due to wide neck of the aneurysm with its multiple collaterals. The patient was re-operated. During the surgery, we observed that the right hepatic artery was found clipped proximal to the aneurysm and feeding of the aneurysm was provided by the left hepatic artery trough multiple collaterals. We ligated the right hepatic artery distal to the aneurysm. Intraoperative Doppler ultrasound was used to decide whether hepatic collateral flow is sufficient or not when aneurismal collaterals are ligated. After clamping the aneurismal collaterals for 15 minutes, hepatic inflow was unchanged by Doppler ultrasound. The collaterals were also ligated without the need of vascular reconstruction. The Doppler ultrasound was repeated on the first and seventh postoperative days and showed normal hepatic inflow with an excluded aneurysm. Patient's condition improved rapidly and he is now disease free during follow up.

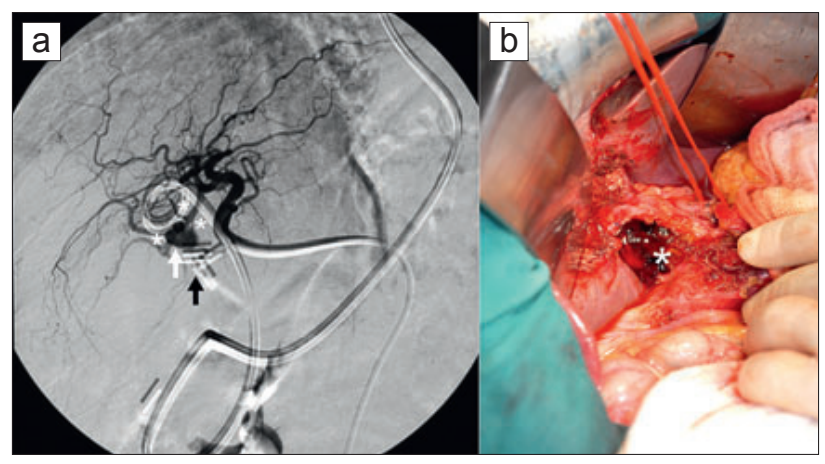

Fig. 2. a) A pseudo-aneurysm just distal to the accidentally ligated right hepatic artery. White arrow shows the aneurysm. Black arrow shows the clips which were placed to the right hepatic artery in the previous cholecystectomy operation. Star shapes show the collaterals around the aneurysm. b) Intraoperative appearance of the pseudoaneurysm within collaterals.

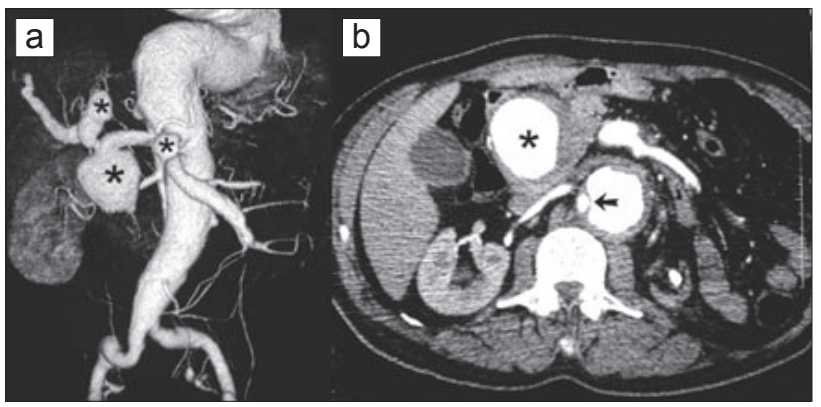

Fig. 3. a) Angiography shows multiple aneurysms originating from common hepatic, right hepatic and superior mesenteric arteries. b) The star shape shows the aneurysm originating from the common hepatic artery. The arrow shows the dissecting abdominal aneurysm.

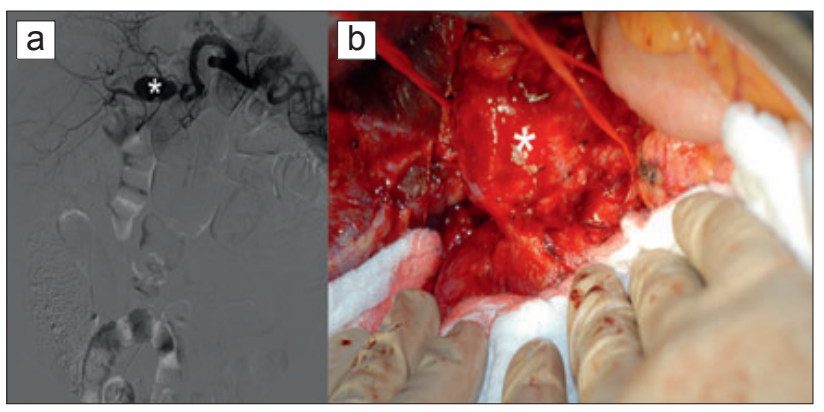

Fig. 4. An aneurysm, localized to the proper hepatic artery. a) Angiographic appearance of the aneurysm. b) Intraoperative appearance of the aneurysm.

\section{Case 3}

A 58-year-old male patient was admitted with complaints of right abdominal pain, jaundice and melana more than one week's duration. Patient's total bilirubin levels were $24 \mathrm{mg} / \mathrm{dl}$ with $18 \mathrm{mg} /$ $\mathrm{dl}$ of direct component. A magnetic resonance cholangiopancreatography $(M R C P)$ was performed and dilatation of the right and left intrahepatic biliary ducts with no visualization of the distal common bile duct was defined. A percutaneous transhepatic cholangiography $(P T C)$ was performed; hemobilia was detected and an external drainage catheter was inserted. A dissecting abdominal aortic aneurysm was found by abdominal computerized tomography-angiography (CT-angio) which began from descending aorta up to $5 \mathrm{~cm}$ distal to the origin of the renal arteries. The dissection fleb extended to the celiac, hepatic and proximal part of the splenic arteries. A 6x6.7 cm measured thrombosed aneurysm originating from common hepatic artery was also found. Furthermore, a fusiform aneurysm of the right hepatic artery measuring $1.9 \mathrm{~cm}$, a fusiform aneurysm of the left hepatic artery measuring $2.1 \mathrm{~cm}$, an aneurysm of the splenic artery measuring $1.3 \mathrm{~cm}$ and a thrombosed aneurysm of the proximal part of the superior mesenteric artery (SMA) measuring $0,8 \mathrm{~cm}$ were detected (Fig. 3). The dissection fleb involved the proximal part of the SMA. An angiography was planned but the patient began to bleed rapidly through the biliary drainage catheter during this period. An emergent abdominal laparotomy was performed and a 9x8x7 cm measured aneurysm with hematoma of the gastrohepatic region was detected. No surgical intervention could be performed due to bleeding of the aneurysm 
in the form of oozing during dissection and the remaining vascular structures were not appropriate for reconstruction due to multiple aneurysms. A covered stent was placed to occlude the aneurysm by endovascular approach on the first postoperative day. But during the angiography, multiple collateral flows were seen to the distal part of the aneurysm. An embolization was applied to the aneurysm and its collaterals. But one day later, persisted collateral flow to the aneurysm was seen by control Doppler ultrasound. The patient did not allow any surgical attempt due to his hypotensive status and died in a few days from multi-organ failure due to sepsis.

\section{Case 4}

A 68-year-old male patient was admitted with complaints of epigastric pain and jaundice last more than two weeks. The ultrasound (US) revealed dilatation of the intrahepatic biliary ducts within a contracted gallbladder and a $5.2 \times 4.8 \mathrm{~cm}$ measured cystic mass beneath the upper edge of the pancreatic head. $C T$-angio showed a $6 \times 7 \mathrm{~cm}$ measured aneurysm originating from the proper hepatic artery and multiple collateral connections from the celiac trunk to the liver (Fig. 4). Covered stent placement was found unsuitable for this patient because of tortuous vessel with a decreased caliper in size due to compression or spasm of the pseudo-aneurysm. We thought that these factors did not allow catheterization and we performed an explorative laparotomy. The collateral flow from the celiac truncus to the hepatic parenchyma was evaluated by intraoperative Doppler ultrasound before dissecting the ligaments and other structures of the hepatoduodenal region. The collateral flow to the liver seemed quite enough even when the hepatic artery was clamped just proximal to aneurysm. Thus, we ligated the hepatic artery just proximal and distal to the aneurysm. Collateral branches feeding the aneurysm were also ligated. After the ligation, hepatic parenchymal circulation was reevaluated by Doppler ultrasound and adequate intrahepatic arterial flow was observed. A cholecystectomy was applied because of the risk of possible ischemic event related to the proper hepatic artery ligation. The Doppler ultrasound was repeated on the first postoperative day and showed exclusion of the aneurysm without leakage. The patient was discharged on the ninth postoperative day and is disease free until now.

Except of the third case, the remaining three patients are alive with normal liver function tests. Control CT-angio was performed for each patient after twelve months and shrinkage of the thrombosed aneurysm with disappearing of compressing sings and normal hepatic flow was observed.

\section{Discussion}

HAA's are rare and usually solitary lesions (90\%), which are more common among males over 60 years. HAA's are localized in the extrahepatic region in $75 \%$ of cases, and from these $63 \%$ involved the common and proper hepatic, $28 \%$ the right hepatic, $5 \%$ the left hepatic and $4 \%$ both hepatic arteries (4). Pseudoaneurysms should be differentiated from extrahepatic aneurysms. Histopathological findings have shown that up to $50 \%$ of HAAs are pseudo-aneurysms (1). This high rate is a natural result of the widespread and increasing usage of invasive percutaneous proce- dures, as well as of $L C s$, which can cause damage to the hepatic artery wall. The occurrence of hepatic artery pseudo-aneurysms after $L C$ was reported as $0.6 \%$ in a series of $1513 L C s$ (5). While pseudo-aneurysms lack of appropriate wall structure or thickness, and carry a high risk of rupture, they should be treated regardless of their diameter (6). HAAs are often asymptomatic unless they rupture. The most common symptom is epigastric or right abdominal quadrant pain. The Quincke's triad including epigastric pain, jaundice and hemobilia are present in up to one third of cases (7). In the present report, two of our patients had epigastric pain with jaundice whereas the other two patients had upper gastrointestinal bleeding.

The progression of HAA's is not well known and mortality rises up to $40 \%$ when spontaneous ruptures occur. Spontaneous rupture occurs more in non-atherosclerotic patients; especially in multiple aneurysms like polyarteritis nodosa. Treatment strategies of HAA are still not standardized and controversies exist regarding the treatment of asymptomatic patients. Indications for the treatment of HAAs are aneurysms $>2 \mathrm{~cm}$, symptomatic aneurysms, non-atherosclerotic aneurysms and multiple aneurysms (4). In the present report, all aneurysms were symptomatic, non-atherosclerotic and bigger than $2 \mathrm{~cm}$. In addition, one patient had multiple aneurysms.

CT-angio is useful for determining the type, number and localization of the aneurysm and its associated collaterals. Therefore, CT-angio should be considered as the first step for optimal treatment planning. Aneurysms can be treated by endovascular embolization, but complications due to embolization have been frequently reported and expanding covered stents began to take its place (8). Our first case had a pseudo-aneurysm localized to the right hepatic artery in whom we successfully used a self expanding covered stent, which provided exclusion of the aneurysm with a patent hepatic artery flow.

Covered stent placement should be considered as the initial treatment of all aneurysms (8-12). Endovascular repair (covered stent placement and/or embolization) was found particularly useful in patients with multiple co-morbidities, in malignancies, and can be used in cases with hemodynamic instability (9). The initial technical success rate for the endovascular procedures was reported as $89 \%$ in a previous study by Sachdev et al (9), who pointed out that secondary interventions for persistent perfusion were successfully performed and failures of primary treatment can often be successfully managed percutaneously.

Embolisation should be avoided when there is not enough collateral flow to feed the liver parenchyma. Otherwise, complications such as hepatic necrosis, liver abscess or cholecystitis may occur. One of the unintended consequences is development of recanalization after embolization (13).

In the present study, covered stent placement was unsuitable in the second and fourth cases due to tortuous and small sized vessels, which were inappropriate for covered stents currently available in the market. In these cases (case 2 and 4), embolization would also not show the expected effect because of wide neck of the aneurysm with its multiple collaterals. Exclusion of the aneurysm was achieved by ligation of the aneurismal collaterals. The third case had multiple aneurysms in the celiac region with concomitant dissecting aortic abdominal aneurysm which had bleeding in the form of oozing and did not allow any surgical intervention. Em- 


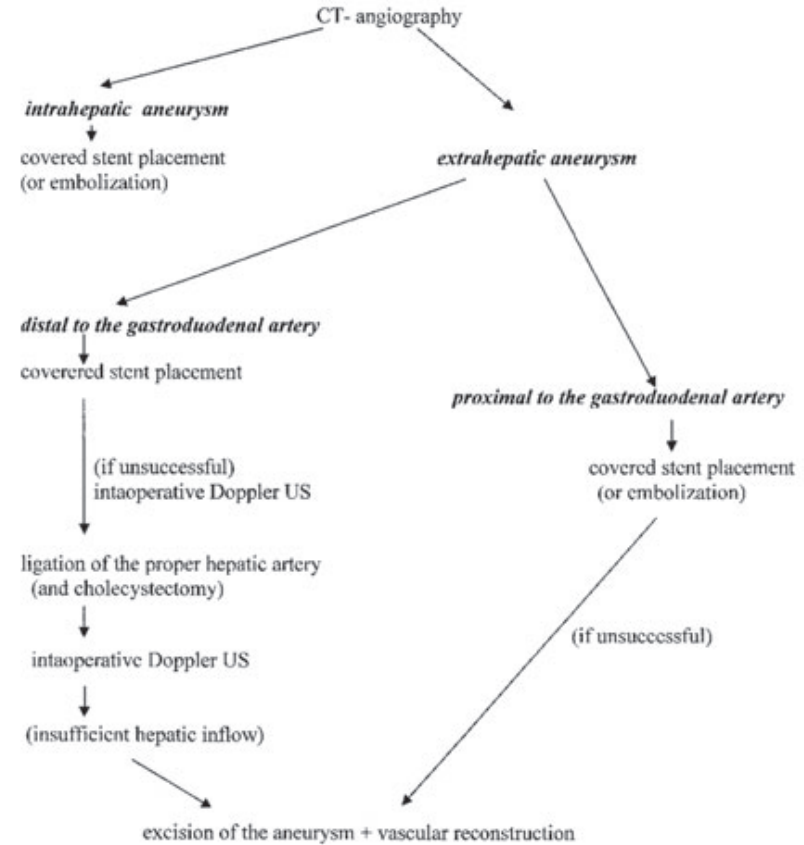

Fig. 5. Treatment algorithm for hepatic artery aneurysms.

bolization was applied by the second angiograpghy after covered stent placement for preventing leakage but was unsatisfactory.

HAAs involving both left and right hepatic arteries can be completely excised and reconstructed with vascular replacement. But this complex method has a high morbidity rate, and liver failure or graft thrombosis is reported after vascular reconstructions (14). Furthermore, vascular reconstruction may not always be needed as seen in our second and fourth cases.

Vascular reconstruction is not usually necessary for aneurysms which are localized proximal to the gastroduodenal artery, because the hepatic flow is maintained through the gastroduodenal arc or other collaterals. However, ligation of the common hepatic artery is contraindicated if occlusion or stenosis exists at the origin of the SMA, because the main source of the small intestine is the common hepatic artery in that cases. For aneurysms localized distal to the gastroduodenal artery, cover stent placement can be preferred initially if appropriate. If not, vascular reconstruction should be considered (1). However, rarely, as in our fourth case, ligation of proper hepatic artery may be sufficient. These circumstances should be confirmed by CT-angio before any intervention.

HAAs may lead to the development of hepatic collateral inflow such as the inferior panctreatico-duodenal arc, left or right gastric arteries, pericholedochal arteries, and arteries from the round or falciform ligaments (15). These collaterals should be demonstrated preoperatively by CT-angio if hepatic artery ligation is planned for aneurysms that are localized distal to the gastroduodenal artery. If the collaterals could not be shown by CT-angio or if there is doubt about the adequacy of the collaterals, the surgical team should be prepared for vascular reconstruction. The intraoperative use of Doppler ultrasound can be helpful at this point to evaluate the hepatic flow and can give valuable information for a decision wheth- er ligation of the proper hepatic artery is sufficient or additional vascular reconstruction is necessary. Cholecystectomy is recommended if proper hepatic artery is ligated to avoid from ischemic events. In the light of the current literature and our own experiences with regard to the treatment options mentioned above, we have developed an algorithm schema for the treatment of HAAs (Fig. 5).

In conclusion, HAA is a rare but more commonly observed clinical entity, which treatment should be handled for each patient separately. CT-angio is useful in determining the type, number and localization of the aneurysm, and should be considered as the first step for treatment planning. Additionally, Doppler ultrasound gives valuable information about the hepatic collateral flow, which facilitates decision making for an intraoperative plan.

\section{References}

1. Alhawsawi AM, Aljiffry M, Walsh MJ, Peltekian K, Molinari M. Hepatic artery aneurysm associated with prune belly syndrome: a case report and review of the literature. J Surg Education 2009; 66: 43-47.

2. Kehr H. Der erste fall von erfolgreicher unterbindung der arteria hepatica propria wegen aneurysma. Munch Med Wschr 1903; 1: 1861-1867.

3. Messina LM, Shanley CJ. Visceral artery aneurysms. Surg Clin North Am 1997; 77: 425-442.

4. Abbas MA, Fowl RJ, Stone WM, Panneton JM, Oldenburg WA, Bower TC et al. Hepatic artery aneurysm: factors that predict complications. J Vasc Surg 2003; 38: 41-45.

5. Nicholson T, Travis S, Ettles D, Dyet J, Sedman P, Wedgewood K et al. Hepatic artery angiography and embolization for hemobilia following laparoscopic cholecystectomy. Cardiovasc Intervent Radiol 1999; 22: 20-24.

6. Tessier DJ, Stone WM, Fowl RJ, Abbas MA, Andrews JC, Bower TC et al. Clinical features and management of splenic artery pseudoaneurysm: case series and cumulative review of literature. J Vasc Surg 2003; 38: 969-974.

7. O'Driscoll D, Olliff SP, Olliff JF. Hepatic artery aneurysm.Br J Radiol 1999; 72: $1018-1025$.

8. Hashim A, Allaqaband S, Bajwa T. Leaking hepatic artery aneurysm successfully treated with covered stent. Catheter Cardiovasc Interv 2009; 74: 500-505.

9. Sachdev U, Baril DT, Ellozy SH, Lookstein RA, Silverberg D, Jacobs TS et al. Management of aneurysms involving branches of the celiac and superior mesenteric arteries: a comparison of surgical and endovascular therapy. J Vasc Surg 2006; 44: 718-24.

10. Aburano H, Morinaga K, Shigenari N, Inoue D, Okuda M, Minami T et al. Common hepatic artery aneurysm treated with an endovascular stent-graft: a case report. Abdom Imaging 2010; 35: 215-7.

11. Pasklinsky G, Gasparis AP, Labropoulos N, Pagan J, Tassiopoulos AK, Ferretti J et al. Endovascular covered stenting for visceral artery pseudoaneurysm rupture: report of 2 cases and a summary of the disease process and treatment options. Vasc Endovascular Surg 2008; 42: 601-606.

12. Balderi A, Antonietti A, Pedrazzini F, Ferro L, Leotta L, Peano E et al. Treatment of a hepatic artery aneurysm by endovascular exclusion using the multilayer cardiatis stent. Cardiovasc Intervent Radiol 2010; 33: 1282-1286.

13. Tarazov PG, Ryzhkov VK, Polysalov VN, Prozorovskií KV, Polikarpov AA. Extraorganic hepatic artery aneurysm: failure of transcatheter embolization. HPB Surg 1998; 11: 55-59.

14. Sessa C, Tinelli G, Porcu P, Aubert A, Thony F, Magne JL. Treatment of visceral artery aneurysms:description of a retrospective series of 42 aneurysms in 34 patients. Ann Vasc Surg 2004; 18: 695-703.

15. Majno PE, Prêtre R, Mentha G, Morel P. Operative injury to the hepatic artery: consequences of a biliary-enteric anastomosis and principles for rational management. Arch Surg 1996; 131: 211-215.

Received April 6, 2011. Accepted August 18, 2012. 